Revue d'histoire de l'Amérique française

REVUE D.HISTOIRE DE L'AMÉRIQUE FRANÇAISE

\title{
BAILLARGEON, Georges-E., La survivance du régime seigneurial à Montréal. Montréal, Le Cercle du Livre de France, 1968. 309 p. \$3.75.
}

\section{Jacques Boucher}

Volume 22, numéro 1, juin 1968

URI : https://id.erudit.org/iderudit/302770ar

DOI : https://doi.org/10.7202/302770ar

Aller au sommaire du numéro

Éditeur(s)

Institut d'histoire de l'Amérique française

ISSN

0035-2357 (imprimé)

1492-1383 (numérique)

Découvrir la revue

Citer ce compte rendu

Boucher, J. (1968). Compte rendu de [BAILLARGEON, Georges-E., La survivance du régime seigneurial à Montréal. Montréal, Le Cercle du Livre de France, 1968. 309 p. \$3.75.] Revue d'histoire de l'Amérique française, 22(1), 128-131.

https://doi.org/10.7202/302770ar d'utilisation que vous pouvez consulter en ligne.

https://apropos.erudit.org/fr/usagers/politique-dutilisation/ 
BAILlaRgeon, Georges-E., La survivance du régime seigneurial à Montréal. Montréal, Le Cercle du Livre de France, 1968. 309 p. $\$ 3.75$.

Monsieur Baillargeon est un spécialiste des problèmes politiques et des mouvements sociaux causés par la survivance en plein XIXe siècle, dans la vallée du Saint-Laurent, d'un système adapté aux exigences du développement et de l'exploitation de la Nouvelle-France, et qui fut maintenu par la politique d'apaisement et de concessions mise de l'avant par Carleton: le régime seigneurial ${ }^{1}$.

Le régime seigneurial canadien avait épuisé ses ressources vers 1820 dans les régions rurales. Depuis fort longtemps, dans les villes, le système faisait figure d'anachronisme, d'entrave au développement économique. L'A. a déjà minutieusement analysé l'important mouvement d'opinions soulevé par le maintien du

$1 \mathrm{M}$ arcel-Joseph, G.-E. Baillargeon, L'abolition du régime seigneurial, 1820-1854, thèse de Ph.D. (histoire), Université de Montréal, 1963; id, Les Canadiens veulent conserver le régime seigneurial, Thèse de M.A. (histoire), Université de Montréal, 1952. 
régime seigneurial, mouvement qui aboutit à son abolition en 1854. On oublie trop souvent que la loi de 1854 ne s'appliquait pas à toutes les seigneuries de la province du Bas-Canada et que celle de Montréal, notamment, ne tombait pas sous le coup de cette loi. Monsieur Baillargeon étudie donc dans l'ouvrage qui vient de paraître, le problème tel qu'il se posait à Montréal, les différentes mesures prises par le gouvernement pour permettre aux censitaires de commuer leur tenure ainsi que l'abolition définitive du régime seigneurial à Montréal en 1859; c'est là l'objet de la première partie de l'ouvrage. Dans la seconde partie, il étudie en outre (et conteste) l'interprétation donnée à la loi de 1859, par les Suplpiciens surtout, interprétation qui a pour effet de maintenir en 1968 un droit de créance apparemment fort rentable à l'égard des successeurs des censitaires qui n'ont pas encore racheté les redevances seigneuriales conformément aux dispositions de cette loi. Comme on le voit, l'A. fait à la fois œuvre d'historien et de juriste; à ce double titre, "La survivance du régime seigneurial à Montréal" intéresse les membres des deux disciplines.

Le sort du régime seigneurial à Montréal depuis la conquête jusqu'en 1859 est intimement lié aux problèmes fort complexes de l'existence légale du Séminaire de Saint-Sulpice de Montréal, donc à la valeur du titre des titulaires de la seigneurie la plus riche sans doute de cette province; l'incertitude devait régner sur cette question jusqu'en 1840. En effet, au moment de la conquête, la seigneurie de Montréal appartenait au Séminaire de Saint-Sulpice de Paris. Les capitulations et le traité de Paris avaient-ils eu pour effet de maintenir dans sa propriété une société religieuse étrangère ? La donation faite le 29 avril 1764 au Séminaire de Saint-Sulpice de Montréal était-elle valide ? La Couronne britannique n'était-elle pas devenue titulaire de cette seigneurie ? La tolérance à l'égard du Séminaire était-elle suffisante pour justifier son titre de propriété contre la Couronne ? Autant de questions sur lesquelles se penchèrent les conseillers juridiques du gouverneur en 1789, auxquelles ils répondirent en niant les droits des Sulpiciens. Cette interprétation fut d'ailleurs confirmée par la Cour du Banc du Roi en 1816 dans la fameuse affaire Fleming: lors d'une contestation au sujet du droit de banalité des Sulpiciens, le tribunal avait aussi conclu à la nullité de leur titre. Depuis ce moment, les seigneurs de Montréal s'abstiendront de porter toute affaire devant les tribunaux et chercheront une solution politique. C'est à cause de la fidélité des Sulpiciens à la Couronne britannique, du rôle qu'ils jouèrent lors des troubles de 1837 que Durham, Colborne et Poulett Thomson 
obtinrent que l'existence légale (donc le titre de propriété) des Sulpiciens soit reconnue par le Conseil Spécial en 1840. La même loi prévoyait en outre les modalités de paiement des arrérages et de rachat des droits seigneuriaux dont le plus lourd, les lods et ventes (taxe de 1/12 du prix de vente en faveur du seigneur), constituait une entrave sérieuse à la libre disposition des biens immobiliers. Le peu d'enthousiasme des censitaires à racheter les droits seigneuriaux, le fait que la loi de 1854 prévoyait une indemnité en faveur des seigneurs à même le trésor public, obligèrent la Législature du Canada-Uni à intervenir en 1859 pour uniformiser la situation à travers le Bas-Canada, pour supprimer les droits seigneuriaux en même temps que le titre de "Seigneur", et transformer certains de ces droits en des créances civiles.

La seconde partie de l'ouvrage qui ne comporte qu'un long chapitre étudie la situation juridique assez trouble à laquelle sont soumis certains propriétaires de Montréal qui se voient à leur grande surprise réclamer le droit de commutation des lods et ventes au tarif le plus élevé prévu par la loi de 1859 et cela sur la valeur acquise par l'immeuble au moment de la transaction. On imagine la réaction d'un propriétaire qui se voit réclamer $1 / 16$ de la valeur présente d'un immeuble qui valait par exemple $\$ 2,000$. en 1859 et plusieurs dizaines de milliers en 1968 ! On comprend également l'indignation de monsieur Baillargeon, qui, après l'analyse des textes de 1859 , conclut à l'illégalité de ces réclamations. Sur ce sujet, l'A. prend à parti le notaire Marler (Law of Real Property) qui s'était porté à la défense des droits des Sulpiciens. Est-il besoin de souligner l'importance économique d'un tel problème ? Souhaitons que les tribunaux du Québec soient saisis de cette question dans le plus bref délai. L'ouvrage de monsieur Baillargeon constituera alors une des pièces les plus importantes au dossier des juristes qui auront à se pencher sur cette affaire complexe. Certes, l'A. n'est pas avocat et son analyse risque de parfois manquer de la rigueur à laquelle les membres de cette profession sont habitués; son mérite, selon nous, ne s'en trouve qu'accru. Puisque nous en sommes au titre des réserves, soulignons que l'énorme documentation dont l'A. fait usage et le souci fort louable d'en faire profiter le lecteur, alourdit le texte au point d'en rendre parfois la lecture difficile. Par ailleurs, devant les exigences abusives des Sulpiciens, monsieur Baillargeon exprime sa désapprobation dans un style lapidaire qui ajoute de la vie à un exposé fort technique mais qui risque de lui faire perdre la sérénité dont certains historiens se font un panache. Cependant, pouvons-nous reprocher à un auteur de se passionner pour son sujet ? Formulons une autre réserve: nous aimerions, 
surtout dans la première partie, que l'A. analyse et critique plus avant certaines questions fort complexes, notamment celle de l'existence légale du Séminaire de Saint-Sulpice de Montréal, après avoir fait état des principaux textes en cause.

En somme, et malgré quelques réserves, cette œuvre historico-juridique se place parmi les plus importantes de la littérature contemporaine sur le régime seigneurial, assise de notre droit de propriété.

Nous terminons en citant les dernières lignes de cet ouvrage:

Quelque paradoxal que cela puisse paraître, le plus grand service à rendre aux Sulpiciens, à l'heure actuelle, c'est de leur dire énergiquement qu'ils ne sont plus seigneurs, que tous les droits seigneuriaux ont été abolis - et non mis en conserve - en 1859, que la prescription, en loi, est une force contre laquelle des mots ne peuvent rien parce qu'elle fait partie de la loi elle-même, et, qu'en réclamant des droits prescrits, ils se préparent un second procès Fleming, suivi peut-être de bien des ennuis, attendu que toutes les personnes qui ont payé, au cours des dernières années, dix fois ou cent fois la valeur d'un droit de commutation prescrit, peuvent se faire rembourser cet argent. Qu'ils ne l'oublient pas, leur inscription "seigneurs de Montréal" sur le mur d'enclos du presbytère Notre-Dame n'est plus qu'un souvenir.

Professeur d'histoire du droit

JACQUES BOUCHER

Faculté de Droit

Université de Montréal 